\title{
The emerging and the spread of the fifth Plasmodium responsible of human malaria: Plasmodium knowlesi
}

\author{
Roberto Manfredi ${ }^{1 *}$, Sergio Sabbatani ${ }^{1}$, Sirio Fiorino ${ }^{2,1}$ \\ From $16^{\text {th }}$ International Symposium on HIV and Emerging Infectious Diseases \\ Marseille, France. 24-26 March 2010
}

\section{Background}

On the ground of the most recent evidences regarding the role of some malaria Plasmodia which have primates as natural reservoirs, the Authors focused their attention on the emerging species Plasmodium knowlesi.

\section{Methods}

Based on the international literature reports, $P$. knowlesi infectious foci have been identified in the past decade in Malaysia, and in particular in the States of Sarawak-Sabah (Malaysia Borneo), and in the Pahang region (peninsular Malaysia).

\section{Results}

The valuable role of molecular biology assays (polymerase chain reaction), performed with specific primers for $P$. knowlesi is underlined, since the traditional hemoscopic examination does not allow to distiguish specific features, especially when $P$. knowlesi is compared with protozoa belonging to the species Plasmodium malariae. Furthermore, malaria caused by $P$. knowlesi may be burdened by a severe and sometimes fatal course, since the clinical presentation and course are more severe compared with those of $P$. malariae, especially due to the extremely elevated levels of parasitemia. The most effective vector for $P$. knowlesi transmission is represented by the mosquito Anopheles latens, which may parasite both humans and primates. Among primates, the natural hosts of $P$. knowlesi are represented by the species Macaca fascicularis, $M$. nemestina, M. inus, and Saimiri scirea.

\footnotetext{
* Correspondence: Roberto.manfredi@unibo.it

${ }^{1}$ Infectious Diseases, University of Bologna, S. Orsola Hospital, Bologna, Italy
}

\section{Discussion}

After remarking the possible, severe prognosis of $P$. knowlesi malaria, we underline the paramount importance of a timely diagnosis and therapy, especially when patients gain access to Western Hospitals, after retrurning from South-Eastern Asia regions, where they eventually practiced excursions and trekking activities in the rain forest. When signs-symptoms suggestive of malaria are recognized in subjects coming back from endemic areas, a rapid diagnosis and treatment are crucial in the management of $P$. knowlesi malaria. In the light of the most recent epidemiological issues, certainly $P$. knowlesi may be added to the list of the known human malaria parasites, which until now included $P$. vivax, P.ovale, P. malariae, and P. falciparum, as the fifth potential human pathogenic Plasmodium. In the next future, an extensive surveillance system and an improved epidemiological control will become needed. Paralleling epidemiological studies and public health implications, a more accurate survey of the clinical features of $P$. knowlesi will be strongly needed, since preliminary data indicate an increased disease severity, associated with a greater level of parasitemia, probably secondary also to the progressive increase of the number of interhuman "passages" of this emerging species of malaria Plasmodium.

\section{Author details}

'Infectious Diseases, University of Bologna, S. Orsola Hospital, Bologna, Italy.

${ }^{2}$ Department of Internal Medicine, Budrio, Italy.

Published: 11 May 2010

doi:10.1186/1742-4690-7-S1-P176

Cite this article as: Manfredi et al:: The emerging and the spread of the fifth Plasmodium responsible of human malaria: Plasmodium knowlesi. Retrovirology 2010 7(Suppl 1):P176. 